\title{
Industrial Implementation of Intelligent System Techniques for Nuclear Power Plant Condition Monitoring
}

\author{
G. M. Westa, S. D. J. McArthur ${ }^{\mathrm{a}}$, D. Towle \\ a Institute for Energy and Environment, University of Strathclyde, 204 George \\ Street, Glasgow, UK, G1 1XW
}

b EDF Energy Barnett Way, Barnwood, Gloucester, UK, GL4 3RS

\begin{abstract}
As the nuclear power plants within the UK age, there is an increased requirement for condition monitoring to ensure that the plants are still be able to operate safely. This paper describes the novel application of Intelligent Systems (IS) techniques to provide decision support to the condition monitoring of Nuclear Power Plant (NPP) reactor cores within the UK. The resulting system, BETA (British Energy Trace Analysis) is deployed within the UK's nuclear operator and provides automated decision support for the analysis of refuelling data, a lead indicator of the health of AGR (Advanced Gas-cooled Reactor) nuclear power plant cores. The key contribution of this work is the improvement of existing manual, labour-intensive analysis through the application of IS techniques to provide decision support to NPP reactor core condition monitoring. This enables an existing source of condition monitoring data to be analysed in a rapid and repeatable manner, providing additional information relating to core health on a more regular basis than routine inspection data allows. The application of IS techniques addresses two issues with the existing manual interpretation of the data, namely the limited availability of expertise and the variability of assessment between different experts. Decision support is provided by four applications of intelligent systems techniques. Two instances of a rule-based expert system are deployed, the first to automatically identify key features within the refuelling data and the second to classify specific types of anomaly. Clustering techniques are applied to support the definition of benchmark behaviour, which is used to detect the presence of anomalies within the refuelling data. Finally data mining techniques are used to track the evolution of the normal benchmark behaviour over time. This results in a system that not only provides support for analysing new refuelling events but also provides the platform to allow future events to be analysed. The BETA system has been deployed within the nuclear operator in the UK and is used at both the engineering offices and on station to support the analysis of refuelling events from two AGR stations, with a view to expanding it to the rest of the fleet in the near future.
\end{abstract}

KEYWORDS: Nuclear; Condition Monitoring; agglomerative hierarchical clustering; rule-based reasoning

\section{Introduction}

As the AGR stations in the UK age, there is an increasing need to understand the condition of the reactor core, the major life-limiting component in an AGR station. Inspections undertaken during routine outages, every two-three years, provide high fidelity information on a limited number of channels. Additional information about core condition can be gained from monitoring data obtained during refuelling operations. These refuelling events occur much more frequently than outage inspections, but the raw data requires significant interpretation effort to provide meaningful results. The application of intelligent systems can aid this process, providing a repeatable and reliable method of automatically assessing refuelling data. In addition, intelligent system techniques can be applied to large volumes of this data to uncover trends, which relate to the age and degradation of the graphite core. These trends can be used 
to supplement existing understanding of the ageing process of nuclear graphite and supports the case for continued and extended operation of the AGR NPPs.

This paper is split into the following sections. Firstly a brief introduction to Nuclear Power Generation in the UK is given along with more technical detail concerning the refuelling process and the associated monitoring data. The ageing process of nuclear graphite, from which the major components of the reactor core are constructed, is also described. The second section deals with the use of intelligent analysis techniques to support various aspects of analysing refuelling data, and how the application of these techniques can also provide valuable understanding into long-term trends in the data. These trends can then support continued operation and lifetime extension of the Advanced Gas-cooled Reactor (AGR) Nuclear Power Plant (NPP) fleet in the UK. The final section describes the industrial implementation of these techniques in a decision support system that aids the analysis of refuelling event data for two NPPs in the UK.

\section{Background}

\subsection{Condition Monitoring of Nuclear Power Plants}

Within the UK, the existing fleet of AGR NPPs are approaching the end of their originally anticipated design lifetimes. Condition Monitoring (CM) of the reactors is playing an increasing role in the continued safe operation of the plant as well as contributing to the safety case made for extended operation beyond the original design lifetimes. This increase in condition monitoring activity generates a large volume data that must be analysed. The expertise required to analyse this data is limited to just a few experts and therefore the use of intelligent system techniques to provide automated decision support ensures that this expertise can be utilised more widely, and that some of the routine, labour intensive analysis can be reduced.

\subsection{Nuclear Power Generation in the UK}

Currently, approximately $20 \%$ of the UK electricity demand is met by generation from NPPs. The vast majority, seven out of the eight NPPs, are the secondgeneration AGR designs. These NPPs are approaching the end of their initially intended design lifetimes of 35 years, though some of them have been granted a lifetime extension of a further 5 years. Part of this process of obtaining a license to operate past the initial design lifetimes is the presentation of a safety case to the regulator, the Nuclear Installations Inspectorate (NII). According to NII technical guidelines (Boyle, 2002), the safety case is described as:

"... the totality of documented information and arguments developed by the licensee, which substantiates the safety of the facility, activity, operation or modification. It provides a written demonstration that relevant standards have been met and that risks have been reduced 'so far as is reasonably practicable' (SFAIRP)"

One method to ensure risk reduction is to undertake analysis of condition monitoring data to provide an improved understanding of the current health of 
the NPP, and in particular the graphite core. The work described in this paper aims to support this goal.

\subsection{Reactor Core Construction}

With AGR nuclear power plants the dominant life-limiting feature is probably the condition of the graphite core. The function of the graphite core is to act as a moderator for slowing the fast neutrons during the nuclear reaction and to provide a structure that allows un-impeded movement of both fuel and control rods as well as adequate cooling of both the fuel and the graphite moderator. The core is constructed from columns of graphite bricks that form vertical channels for fuel assemblies, control rods, instrumentation channels and coolant flow. Though the exact configuration changes from station to station, approximately 19,000 bricks comprise the core, which are spread over 12 layers, resulting in over 300 fuel channels per core. Figure 1 shows a photograph showing the arrangement of the graphite fuel bricks during construction. The material properties of graphite change due to neutron irradiation and radiolytic oxidation encountered in the reactors, during normal operation. This affects the dimensions, internal stress and integrity of the graphite bricks, which in turn could impede the movement of fuel, control rods and coolant through the core. Knowledge of the current dimensions of the graphite bricks that comprise the core is therefore a key requirement of understanding the current condition of the core. These dimensions are routinely obtained from inspecting the core during outages. Typically, every three years a reactor will undergo an outage where a limited number (currently 31 for the oldest reactors, though much less for the younger reactors) of fuel channels are inspected. Inspection includes visual inspection of the channel walls using special TV camera equipment, accurate measurement of the diameter and tilt of the channel bore across the fuel height of the channel, and trepanning small samples of the core which are then subject to a series of material properties tests. Selection of these channels for inspection is undertaken to ensure a representative subset of the whole core is obtained, as well as targeting individual channels which may contain known issues. The inspection campaign provides very detailed information on the health of the core, but based on a limited number of channels.

As the nuclear power plant ages, there is a greater pressure to increase the volume and periodicity of these inspections. Inspections are costly, however, as the reactor must be offline (and thus not generating electricity) and the fuel channel to be inspected temporarily emptied of fuel. Limitation on buffer storage space restricts the number of channels that can be empty at a given time and movement of fuel between the reactor and the store is a time consuming process. One approach to mitigate the need for some of the increased inspections is to increase the amount of online monitoring that is undertaken. One existing source of monitoring data that can provide information relating to the current channel dimensions is fuel grab load trace data, gathered during refuelling operations. This condition monitoring data needs to be analysed to provide meaningful results, which is a labour intensive process requiring specialised knowledge. IS techniques have been successfully applied to address this issue and is a key contribution of this work. 


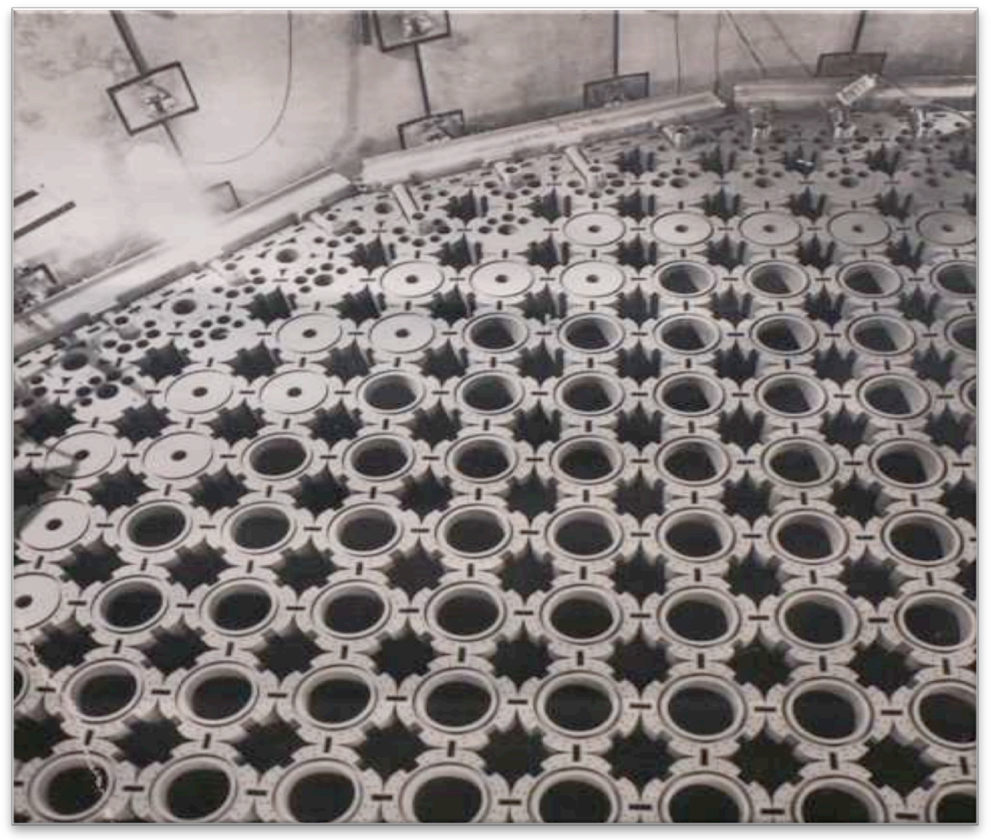

Figure 1. Photograph showing the layout of the reactor core during construction showing the arrangement of a layer of graphite fuel bricks. Picture courtesy of EDF Energy Ltd.

\subsection{Fuel grab load trace online monitoring data}

AGR stations are refuelled on a regular basis, with the fuel for a single channel lasting approximately 6 to 7 years. The uranium fuel is housed within refuelling assemblies that are inserted into a fuel channel that has been evacuated during the refuelling process. Typically the reactors are refuelled in batches, with 8-10 fuel assemblies being exchanged every 6-8 weeks (Ziver et al., 2004). A full description of the fuel assembly can be found in (Ng, 1995), but the point of interest here is that the fuel assembly contains sets of stabilising brushes that guide the fuel assembly through the core. As the fuel assembly is lowered into the core, these brushes form an interference fit with the fuel channel wall, and as a result a friction force is generated. During refuelling a load cell records the apparent weight of the fuel assembly along with a measurement of position into the core. Changes in the dimension of the channel, such as those caused by known features such as the piston seal bore, the guide tubes and the stand pipes, as well as changes caused by ageing and distortion of the graphite itself will result in a change in the frictional component of the measured load. The measured load over the course of a refuelling operation is termed the Fuel Grab Load Trace (FGLT) and this can provide information relating to the current health of the core. These FGLT traces are manually assessed by an expert who has built up an understanding of what constitutes a normal FGLT through years of experience, supported by a theoretical understanding of the refuelling process and experimental rig-work and simulation to generate profiles of anomalous behaviour (Roscow et al., 2008). Following every refuelling campaign, these FGLT are manually analysed and the results are fed into a quarterly Monitoring Assessment Panel (MAP) meeting, where the results of analysis are compared with other sources of monitoring data such as thermal to neutron power ratios (Haddock \& Parks, 1995) and control rod movements (Wallace et al., 2010) to 
determine whether there is any indication of anomalous behaviour which might suggest core distortion.

Two issues with the manual analysis of FGLT are:

1. The paucity of expertise. Knowledge and understanding of what constitutes a normal FGLT and how anomalous core behaviour may manifest itself in the FGLT is limited to only a few experts. As the volume of condition monitoring data that is required to be analysed increases, a greater burden is placed on this limited resource.

2. Manual analysis is based on human judgement. Different experts may interpret the raw data slightly differently. Though anomalous behaviour is discussed with a wider pool of expertise, conflicts may arise particularly with borderline cases.

The application of intelligent system techniques addresses these two issues by providing a captured and encoded version of the human expertise which can be applied to provide a repeatable and auditable diagnosis of FGLT data, thus supporting, but importantly not replacing, the decision process of the human expert.

\subsection{Related work}

The application of intelligent systems to problems in the nuclear domain is well reported, for example (Beck \& Behera, 1993) reports over one hundred applications of expert systems to the nuclear domain, though many of the articles describe proposed concepts and prototype systems rather than industrial deployment. None of these relate directly to graphite core condition monitoring and the analysis of FGLT, though previous work has seen the development of ALTA, an intelligent system for the analysis of FGLT to determine whether fuel has set down correctly in the bottom of the core (Steele et al., 2003). ALTA used a combination of C4.5, Kohonen Networks and K-means clustering to establish the location of the fuel touchdown point and has implemented a rule-based system to assess the set down process to ensure that each stage has been completed successfully. This work established that FGLT could be analysed to provide information relating to fuel set-down, the research presented herein expands on these concepts to provide additional information relating to the core health. In the wider field of providing information relating to core health, control rod performance data has been examined (Wallace et al, 2010) to identify potential issues with the core while (Jahn, et al., 2007) describes the IMAPS system which supports station Monitoring Assessment Panel (MAP) meetings. The MAP meetings are designed to assess all relevant condition monitoring data which may indicate core distortion over a given time period. These meetings normally take place quarterly and include assessment of the results of FGLT analysis in the context of reactor wide monitoring. This represents the extent of work undertaken which applies intelligent analysis techniques to the analysis of condition monitoring data for AGR stations and the contribution provided by this work demonstrates the use of IS techniques to provide timely and repeatable analysis of FGLT data to support continued and 
extended operation of the AGR NPPs, augmenting the existing manual analysis currently undertaken.

\section{Automated Analysis of FGLT}

The approach adopted was to understand how the refuelling events were manually analysed and identify parts of the process that could be supported through the application of intelligent analysis techniques. This manual analysis process consists of plotting the raw data signal, visually determining the location each of the brick layers through identification of the brick interfaces and assessing the response for each individual brick interfaces. This assessment is based on knowledge of previous defect free responses and simulated responses of potential defects obtained from experimental rig work. The experimental understanding of brick defects had been built up based on a general model of any brick, rather than specifically tied to a layer in the core. It was therefore important to maintain the approach of segmenting the data into individual brick layers. A rule-based approach, rather than an algorithm for detecting the brick interfaces was selected for flexibility and for explicability of results. Furthermore, this approach meant that the system could be easily configured for use at different stations, the rules about what constitute an interface are generic to all stations, but the exact sizes and limits are individual to each station instance. Similarly a rule-based approach was chosen to represent the knowledge of defects, again to allow flexibility whereby limits could be tailored to individual stations, new defect shapes could be easily added, and also so that the results could be easily explained through presentation of the rules which had fired for any given case. In order to detect where anomalous behaviour was present, but not attributable to known defect responses, an expert assesses the data based on his/her understanding of what a normal response should be. To undertake this process in software, the data from each brick layer is compared with a user-defined benchmark of normal behaviour, to assess whether it contains anomalous behaviour. The application of clustering algorithms provides support to the user when defining these benchmarks. These clustering techniques provide a visual means of ensuring that the benchmark data against which new events are assessed is suitable. It is possible for the reactor to be refuelled at different operating conditions, which leads to slightly different normal responses. It would be unsuitable to combine both of these responses together when defining a benchmark, so clustering helps the user to group like responses together. Furthermore, it would be unsuitable to define a benchmark of normal behaviour, which contained instances of anomalous behaviour. Again, clustering techniques can help to identify these outlying anomalous events. Finally, the core is ageing all the time and as a result the normal benchmark is also changing. The final application of intelligent systems techniques explores long-term trends within the data and how automated learning can support the definition of future benchmark envelopes. These four applications of intelligent analysis techniques are now described in further detail. 


\subsection{Expert System for feature identification}

\subsubsection{Overview}

The raw FGLT data generally follows a repeatable pattern between refuelling events and across different channels. Figure 2 shows multiple refuelling events plotted on the same graph, the upper set of traces originate from removal of old fuel form the core while the lower set of traces originate from the insertion of new fuel into the core. As can be seen in the plot, the traces from the same set follow a repeatable pattern across different refuelling events.

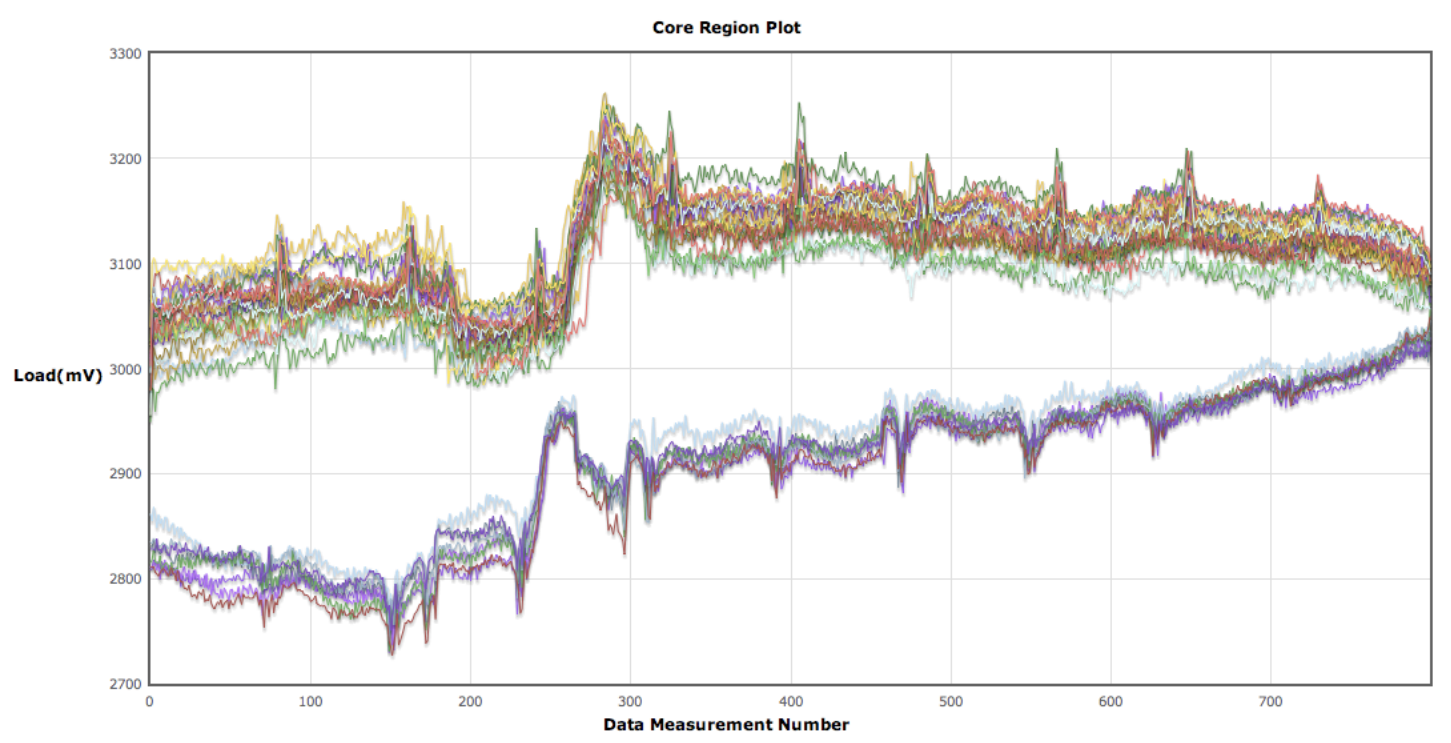

Figure 2. Plot showing multiple FGLT traces on the same graph, illustrating the repeatability of the response between different events

The measured load which comprises the FGLT consists of a number of components, namely the absolute weight of the fuel stringer, effects of cooling gas flowing through the channel and around the fuel assembly which supports the weight at certain points, and a frictional component caused by sets of stabilising brushes interacting with the channel wall to guide the fuel assembly through the core. At various points through the fuel channel the bore diameter changes, for example, the guide tube section that sits on top of the graphite core is of a larger diameter then the bore diameter of the graphite bricks. At start-oflife, when the graphite bricks are un-irradiated, the graphite core is straight. As the graphite ages, there is a tendency for the bricks to 'barrel', with the top and bottom of the brick shrinking more than the centre. This results in a narrower bore at the brick interfaces, which manifests as a peak in the FGLT. Identification of these interfaces is a primary goal of the feature identification expert system.

\subsubsection{Approach}

The approach to identifying the brick interfaces is as follows. Firstly, the raw data is converted into a series of peak objects. The FGLT sometimes suffers from noise, and therefore the raw data is assessed to determine whether smoothing is required. The measure of noise is simply the number of peak objects identified in the core region. If too many peaks are present then a 10 point moving average smooth is applied to the data and the peak identification algorithm re-run. The smoothing operation is repeated until sufficiently few peaks are identified, or an 
upper limit of number of smoothing operations has been reached. Each peak is assessed using a forward chaining rule-based expert system that examines the shape, height and relative position of the peaks within the trace to identify a candidate set of peaks, which represent the brick interfaces. A generic rule based is used for all stations, but the generic rules are merged with station specific limits to provide a set of station specific rules for each station. For example, the top of brick layer 5 at Hunterston B Power station may be at a different height from the top of brick layer 5 at Hinkley Point B, but brick layer 6 will always be directly above brick layer 5 .

\title{
3.1.3. Example Rule
}

The following is an example rule taken from the rule base which is used to classify a peak based on its height measurement.

\section{IF}

\author{
THEN \\ [peakHeight] $<$ [BLI 5 to 6 Height Upper Limit]
}

[peakHeight] $>$ [BLI 5 to 6 Height Lower Limit]

AND

[Peak Height Classification] $=$ BLI $5 \rightarrow 6$

The variable peakHeight is tested for the current peak. The limits BLI 5 to 6 Height Lower Limit and BLI 5 to 6 Height Lower Limit are read in from a configuration file specific to the station, thus keeping the station specific limits independent from the generic analysis rules. The rules themselves are stored in an $\mathrm{xml}$ format and can be parsed along with an appropriate limit file to generate a human-readable html web page. This permits the rules used by the expert system to be readily viewed and verified by a human expert, without the need to be versed in the mechanics of the rule-based reasoning engine.

\subsection{Fuel Grab Load Trace Analysis}

\subsubsection{Overview}

Once the brick interfaces have been identified then the data associated with each brick layer can be separated out. When manually assessing FGLT traces, the engineer will use his/her knowledge and expertise in assessing whether the shape of each brick layer is normal. This knowledge and expertise is built up from many years of assessing FGLT data coupled with a strong theoretical understanding of how the graphite ages (Brocklehurst \& Kelly, 1993, Marsden et al., 2008, Neighbour, 2000, Heggie, et al., 2005). In addition, inspection data (Cole-Baker \& Reed, 2005) has been compared to the FGLT to understand how some known cracks manifest themselves in FGLT. Furthermore, some of the anomalous behaviour, such as certain types of crack configurations have never been observed in situ (and at this stage are only hypothesised so may never occur). In these situations, experimental rig work has been undertaken and reported in (Roscow, et al., 2008 and Mclachlan, et al, 2005) to generate expected FGLT responses for a wide range of types of crack and configuration. 


\subsubsection{Anomaly detection}

This understanding of normal behaviour has been captured and implemented within BETA. The first assessment that is undertaken is to compare the FGLT under investigation with a benchmark of expected behaviour. This benchmark of expected behaviour is based on empirical data and is created and validate by an engineer in advance of the analysis. The engineer creates a collection of historical FGLT traces that he/she knows is free from defects and anomalous behaviour. The data from this collection is then used to generate an average, or expected response on a brick layer by brick layer basis, along with a maximum and minimum bounded envelope. The FGLT under investigation is then compared, layer by layer, against these envelopes. The measures used to determine the presence of an anomaly are whether the current FGLT exceeds the maximum or minimum bounds, and for how long, as well as simple statistical mean squared error comparison of the new trace with the average trace. This analysis only provides an indication of whether the behaviour appears anomalous or not. This process of defining a benchmark of normal behaviour is supported by hierarchical clustering.

\subsubsection{Hierarchical clustering to support benchmark definition}

The benchmark analysis is based on empirical evidence, using the premise that if a new event is similar enough to previous normal behaviour then it too should be normal. When defining these benchmarks the engineers want to be sure that the source data is sensible and does not contain anomalies. A useful check is to visualise these in a hierarchical manner. Dendrograms (Gower \& Ross, 1969) are a visualisation tool for agglomerative clustering permit the rapid identification of unusual traces. Figure 3 shows a typical dendrogram generated from a collection of FGLT data. In this instance, the collection contains 70 discharge refuelling events and brick layer 6 data is shown. 


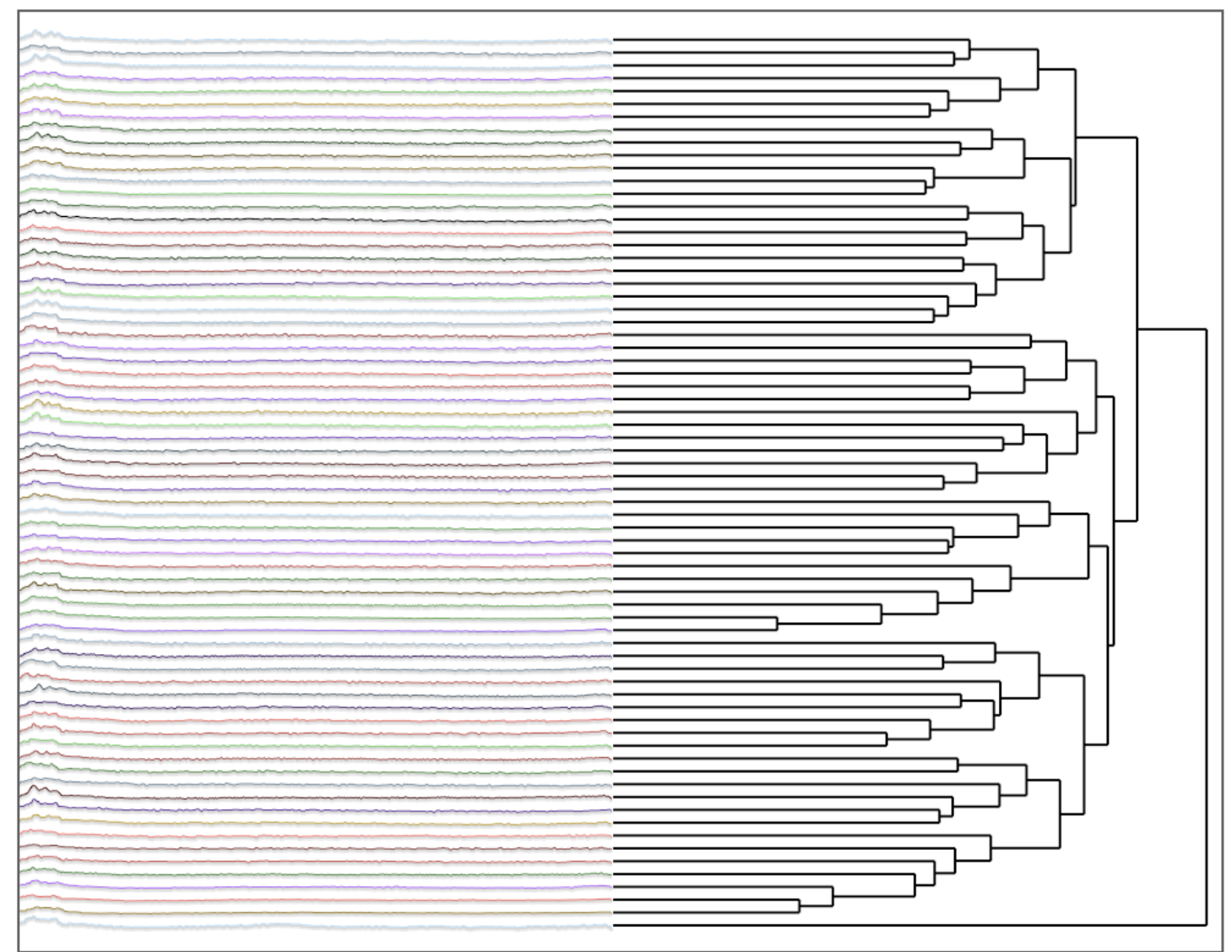

Figure 3. Dendrogram of 70 sets of Brick Layer 6 data using an $\mathbf{r}^{2}$ measure of similarity and the average linkage method. The data has been rescaled so each event is represented by 400 points but the first and last 50 points are omitted from the comparison as they contain the interfaces

Figure 4 shows a zoomed in region of the dendrogram containing the bottom four traces. It can clearly be seen that the bottom most trace contains an addition peak, which was subsequently determined from inspection data to result from a circumferential crack towards the centre of the brick.

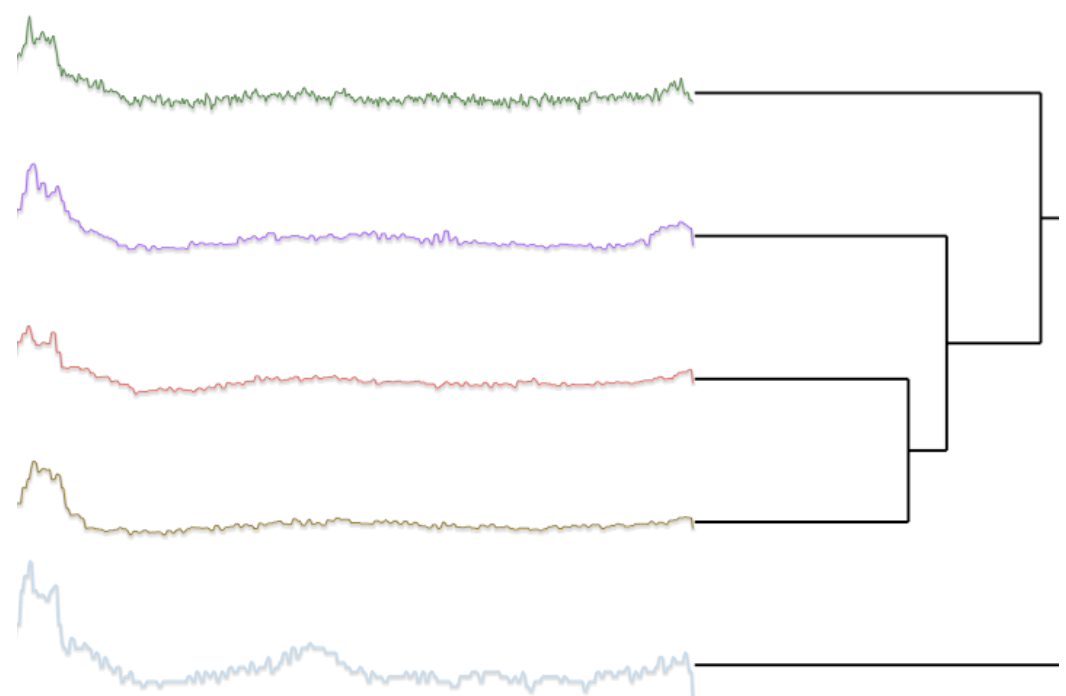


Figure 4. Zoomed in section of the dendrogram showing the bottom 5 traces. The bottom trace is clustered apart from the other traces and exhibits behaviour of a circumferential crack

In this example the measure of similarity between the events is a simple $\mathrm{r}^{2}$ error calculation. More complex measures of similarity could be applied, but for this instance this measure of similarity was sufficient. In addition, the type of linkage used here was average linkage method, though other types are available (Gower \& Ross, 1969).

\subsubsection{Expert System for Fault Classification}

The second assessment which is undertaken is to assess the shape of the brick layer response against idealised responses generated from the experimental rig work. This allows classification of particular expected fault responses to be undertaken. For each brick layer, five key points have been identified, as shown in Figure 5. These points are automatically identified by the feature identification expert system, described earlier, together with the brick interfaces.

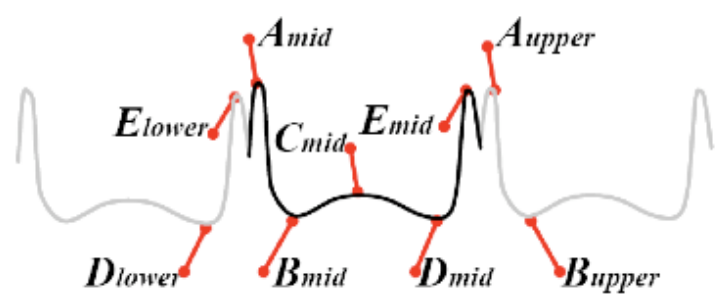

Figure 5. The key points identified for analysis of a brick layer

Using these five points, plus some of the equivalent points from the neighbouring bricks, a set of rules based on calculated values can be generated. For example, a circumferential crack will manifest itself as an additional peak or peaks towards the centre of the brick. An example taken from an actual circumferential crack can be seen in Figure 6, where the smoother line represents the expected normal response, and the line with the large variations towards the centre represents cracked brick behaviour.

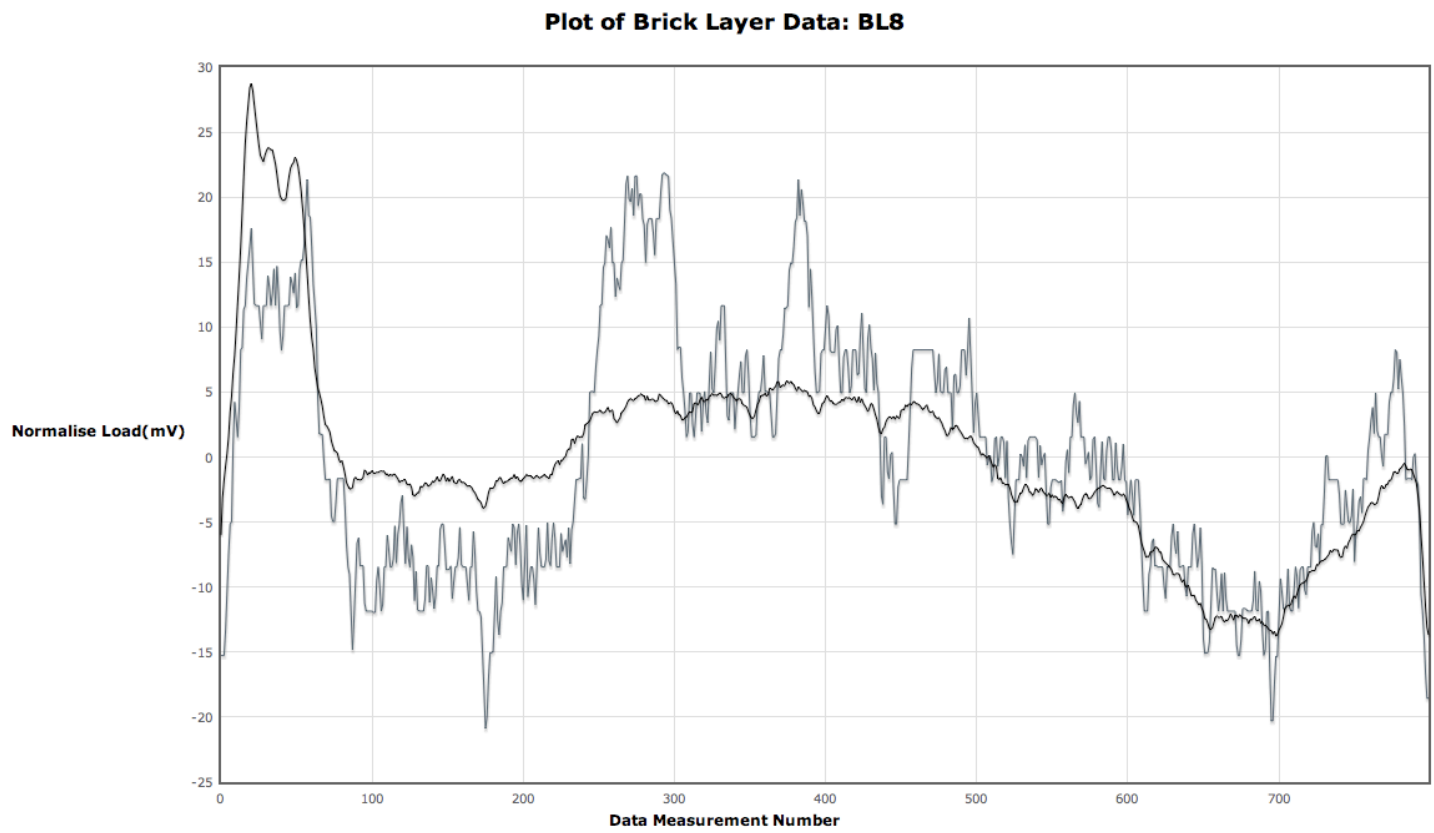

Figure 6. The expected response compared with the response from a cracked brick 
The presence of a circumferential crack can be determined by observing a difference between $C_{\text {mid }}$ and $B_{\text {mid }}$ and a difference between $C_{\text {mid }}$ and $D_{\text {mid }}$ which are higher than expected. Again a measure of what is an acceptable range of values for normal behaviour is required, and as with the envelope analysis described earlier, this can be determined by using historical data. As with defining the benchmarks of normal behaviour, the user selects a collection of historical events as the seed data. Examining the spread of the calculation values provides an indication of the distribution of the values. It is important that the distribution approximates to a uni-modal distribution and does not contain any significant outliers. Indeed this process can be used to determine the presence of possible anomalous behaviour, which should not be included within the benchmark definition. The limits are taken as the maximum and minimum values from the spread, though another statistical measure such as the standard deviation could be chosen instead. To assess a brick layer for the presence of all currently hypothesised cracks, 9 such calculations have been identified. A set of thresholds for each calculated value is required for each brick layer, and each fuel channel has typically 11 layers, resulting in 99 thresholds which must be set for each benchmark. This necessitates using a standard measure such as the maximum and minimum value for each distribution, rather than manually determining each threshold. This process is automated whereby these distributions are automatically generated and through an iterative process the user can remove anomalous events from the collection until a suitable final selection of events is determined. The determination of these benchmarks is critical as they provide the representation of normal behaviour against which new events are compared.

\subsection{Long term trending of data}

The application of these techniques is applied to a system, which is continually receiving new data as the NPPs are refuelled. Over time, it is expected that the M-shaped profile of the brick, shown in Figure 5 will become more pronounced as the graphite ages and distorts. This may then require both the envelopes of expected behaviour and the thresholds used in the classification expert system to be re-defined periodically. When establishing new limits it is important to be able to understand and justify why new limits are required. The BETA system supports this engineering task by providing a means to visualise trends in any of the thresholds mentioned previously through three standard plots which show the variation in data across the core, by using a 2-dimensional core map plot, temporal variation, by plotting the variation in threshold with time and a simple density plot, which shows whether a single or multiple distributions are contained within a single collection of data. Figure 7 shows an example distribution for the distribution of the parameter which measures the magnitude of the peak in the centre of the brick (described in the previous section). In this example, one value is significantly larger than the set, and is highlighted in the core plot and the temporal plot. In the distribution, the single value causes the distribution to have an increased right hand tail. Examining the data associated with this value in isolation reveals an abnormal shape, as shown in Figure 6, thus indicating that this event should be removed from consideration when setting the thresholds for this parameter. 

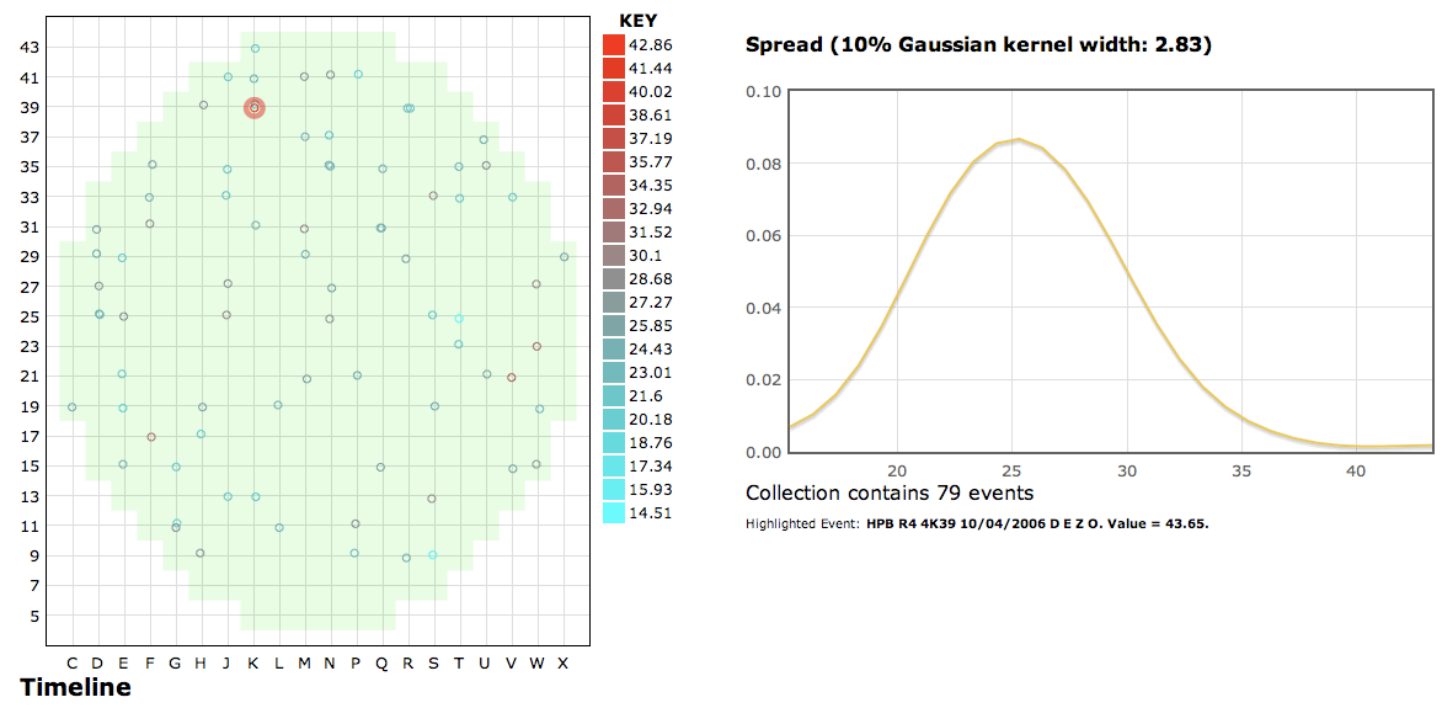

Highlighted Event: HPB R4 4K39 10/04/2006 D E Z O. Value $=43.65$.

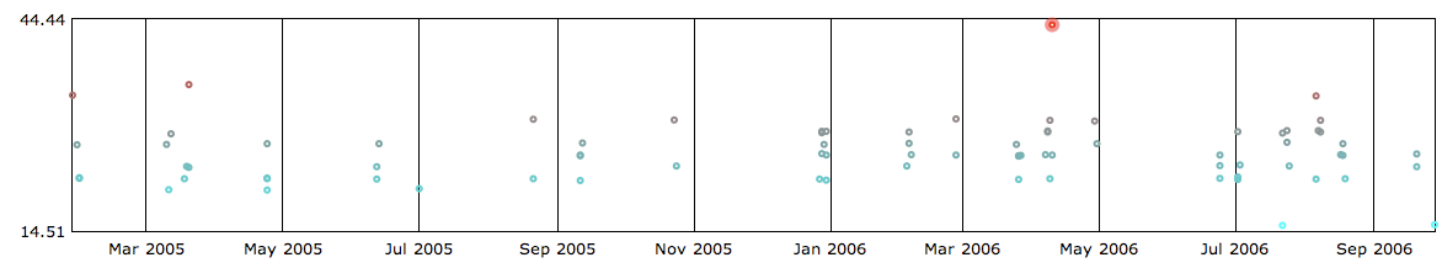

Figure 7. Example showing the distribution of a base calculation, brick layer 8, base calculation 9. An unusual trace is highlighted in red.

This approach can also be used to track the evolution of these limits with time. The example provided in Figure 7 involves a single years worth of data. This time frame is not sufficiently long for a significant change in this parameter value caused by ageing. However, longer time frames will result in this parameter increasing, or in other words as the graphite ages, the brick distortion becomes greater. There will come a point where the limits will need to be adjusted to take into account this change and a temporal plot such as the one shown above, will allow the user to track this change.

\section{Implementation: The BETA System}

\subsection{Approach}

The techniques described in the previous section have been combined and implemented in an industrial strength system, BETA, which is used by Graphite Core Engineers to routinely assess every refuelling event undertaken at two of the AGR stations within the UK, with a view to rolling out to the other stations within the fleet in the near future. A structured approach to the design and development of the BETA system software was undertaken. Using the V-model approach of software engineering (Forsberg \& Mooz, 1992), the necessary user requirements, system design specification and test documentation was produced to ensure the software met the necessary requirements for providing decision support to a process to support a safety case. The system itself was first installed as a stand-alone prototype system presented to a limited number of experts before development as a web-based system accessible throughout the organisation, both at the engineering offices and also from the NPPs themselves. 


\subsection{System Architecture}

The BETA system has three main components, the database, a web-based user interface and the monitoring and analysis engine, as shown in Figure 8.

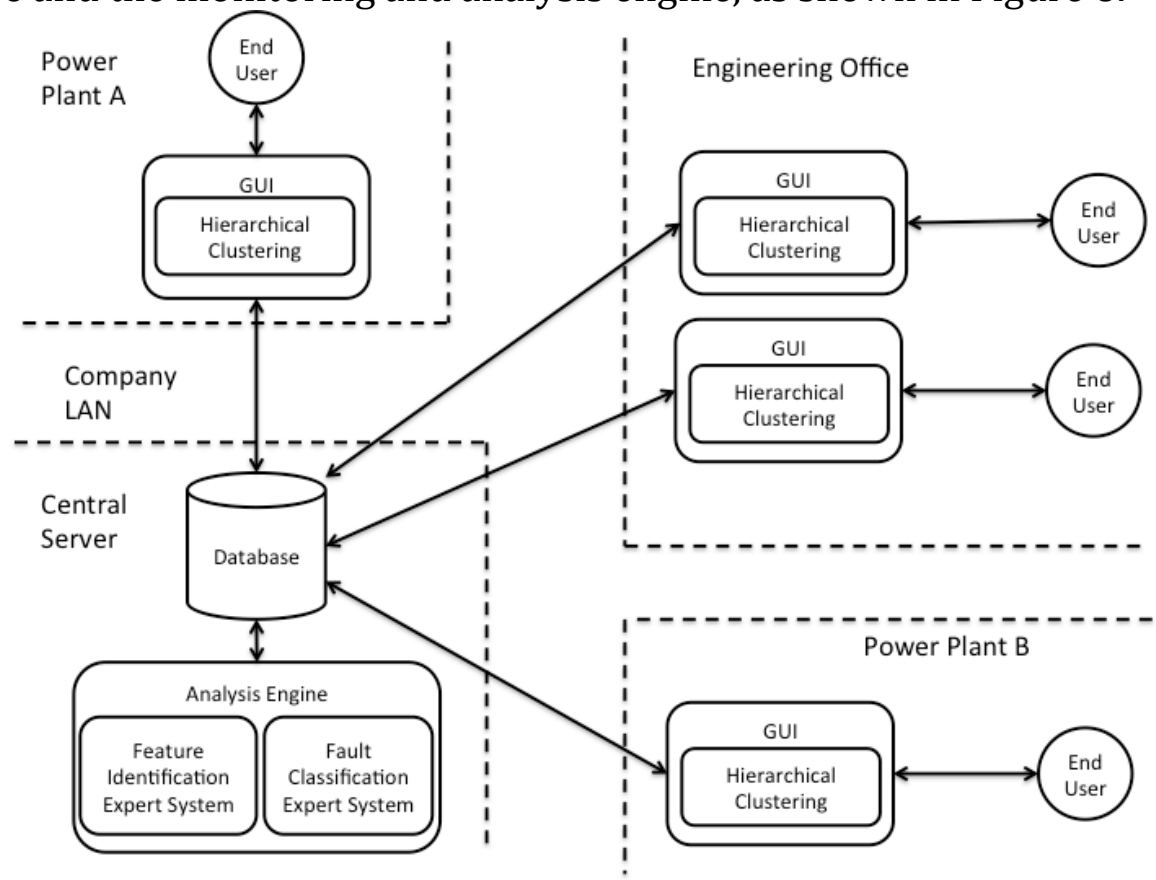

Figure 8. Architecture of the BETA System

\subsubsection{Web-based user interface}

The user interface consists of dynamically generated web-pages. This acts as the interface between the database and the engineer allowing them to perform the following functions necessary for the analysis of FGLT:

- Access any FGLT refuelling event data currently in the database

- Upload new FGLT refuelling event data to the database and subsequently verify any FGLT in the database

- Request the automated analysis of any verified FGLT event within the database, using any pre-defined set of benchmark behaviour

- Define a new benchmark set of behaviour

- Perform data mining on the FGLT data to uncover new patterns

\subsubsection{Monitoring and analysis engine}

The intelligent analysis engine is a stand-alone program that runs as a service on the server along side the database. It constantly monitors the database for new FGLT refuelling data that has been uploaded and for requests to analyse refuelling events. When a new FGLT is detected, it automatically attempts to determine key parameters such as the brick interfaces using the feature identification expert system. The results of the feature identification are stored in the database for use later by the fault analysis expert system. When a new request to analyse a refuelling event is made, the user also selects which predefined benchmark of expected behaviour to use. The request is added to the job queue and analysed when the fault analysis expert system is next available. Once the analysis is completed, the user can access a summary report detailing the results. 


\subsubsection{Database}

An important benefit of this research was to establish a single source of information on fuel grab load trace data. Historically, the FGLT data was captured using a pen and paper plotter. The rolls of paper containing the traces are archived as part of the station records. Though the stations still use the pen and paper plotters, many of the stations were retro-fitted with electronic data capture devices. There is no requirement, however, to archive the electronic data. The NPP engineers transfer the data from the fuelling machine to the company LAN, where graphite core engineers access and analyse the data. The results from the manual analyses are recorded in the company document management system, but there is no formal archive of the raw data. By creating a database of the refuelling data, engineers can rapidly access historical data and undertake data mining to uncover longer-term trends across many refuelling events. A further important consideration was to ensure that the proper quality assurance grade of the data was maintained. To enable this, all data entered into the database must be validated by a second engineer before any analysis can take place. This is an important issue, particularly when in future decisions relating to the continue operation of a station may be made based partly on the data contained within the database. In addition to the raw data, the database also acts as a repository for the results of feature identification from the raw data and as a store for all the results of the analyses undertaken on the data. Finally, the database maintains the status of each analysis request and provides the conduit between the engineer requesting analysis of an event and the automated analysis engine delivering the results to the user.

\subsection{Industrial Deployment}

The BETA system has been installed at EDF on the company LAN. It is accessible by members of the Graphite Core Project team who use the software to support the analysis of all FGLT data arising from two out of the seven AGR NPPs currently operating in the UK. The BETA database has been populated with data gathered from refuelling events since 2003 and currently contains over 1200 individual refuelling events. The system is running concurrently with the manual analysis process, to ensure that the results are consistent with the findings from manual analysis, with the goal of migrating to use of the system as a first point of call for all analysis associated with the FGLT data. To date, the system has been successfully used to identify channels containing defects, which have subsequently been confirmed through later inspection. During operational use, the end users have provided feedback on the system in terms of suggested functionality, and two iterations of the software have been formally released, with a third version currently in development.

\section{Conclusions}

This paper has described the application of intelligent systems techniques to refuelling data gathered from Nuclear Power Plants in the UK to provide automated decision support. The analysis of FGLT data is important as it augments the existing programme of in-core inspections by providing more frequent information relating to reactor core condition. Intelligent Systems techniques provide support to existing manual analysis by providing an 
automated, repeatable and auditable means of assessing the data and thus reducing the reliance on individual experts by making their expertise available within the developed system. Intelligent Systems techniques have been shown to provide automated analysis of new FGLT events, but also support the definition of benchmarks of normal behaviour through the use of clustering algorithms. This allows relevant benchmarks to be defined and maintained, ensuring that the system can adapt to the degradation process of the core. Furthermore, the techniques have also been shown to support the identification of long-term trends in the data. The resulting industrial implementation of these intelligent systems techniques, BETA, is used on a regular basis by graphite core engineers to support the analysis of all refuelling data for two AGR plants in the UK.

\section{Acknowledgements}

This work was funded by EDF Energy. The views presented here are the views of the authors and do not necessarily represent the views of EDF Energy.

\section{References}

Beck, C. E. \& Behera, A. K. (1993) "A Bibliography of Technical Papers Relating Expert Systems to Nuclear Power Plants", IEEE Transactions on Energy Conversion, Vol 8., No. 1, 1993

Boyle, C. (2002) "Guidance on the purpose, scope and content of nuclear safety cases", Health and Safety Executive Report Number T/AST/051, http://www.hse.gov.uk/foi/internalops/nsd/tech_asst_guides/tast051.pdf

Brocklehurst, J. E. \& Kelly, B. T. (1993) "Analysis of the dimensional changes and structural changes in polycrystalline graphite under fast neutron irradiation", Carbon, Vol 31 Issue 1, 155-178

Cole-Baker, A. \& Reed, J. (2005) "Measurement of AGR Graphite Fuel Brick Shrinkage and Channel Distortion", Ageing Management of Graphite Reactor Cores, Cardiff, $28^{\text {th }}-30^{\text {th }}$ November 2005

Forsberg, K. \& Mooz, H. (1992) "The Relationship of System Engineering to the Project Cycle", Engineering Management Journal, Vol. 4, No. 3 September 1992, pp. 36-43

Gower, J.C. \& Ross, G. J. S. (1969) "Minimum Spanning Trees and Single Linkage Cluster Analysis", Journal of the Royal Statistical Society. Series C (Applied Statistics), Vol. 18, No. 1, pp54-64

Haddock, S. A. \& Parks, G. T. (1995) "AGR Fuel Management using PANTHER", Fuel Management and Handling, British Nuclear Energy Society, London, Great Britain, pp. 11-18, 1995 
Heggie, M., Suarez-Martinez, I., Savini, G., El-Barbary, A., Ewels, C. \& Relling, R. (2005) "Irradiation Damage in Graphite from First Principles", Ageing Management of Graphite Reactor Cores, Cardiff, 28 ${ }^{\text {th }}-30^{\text {th }}$ November 2005

Jahn, G. McArthur, S. Reed, J. \& Towle, D. (2007) "Staged Implementation of an Agent Based Advanced Gas-Cooled Reactor Condition Monitoring System," Power Engineering Society General Meeting, 2007. IEEE , vol., no., Pages 1-4, 2428 June 2007

Marsden B J, Hall G N, Wouters O, Vreeling J A \& Van Der Laan J. (2008) "Dimensional and material property changes to irradiated Gilsocarbon graphite irradiated between 650 and 750 C.", Journal of Nuclear Materials. Vol. 381. Issue $1-2,62-67$

Mclachlan, N. Shaw, D. J. \& Salih, H. (2005) "Application of Whole Core Modelling Methodology to Life Extension of AGR Reactor Graphite Cores", Ageing Management of Graphite Reactor Cores, Cardiff, 28 ${ }^{\text {th }}-30^{\text {th }}$ November 2005

Neighbour, G.B. (2000) "Modelling of dimensional changes in irradiated nuclear graphites”, J. Phys. D: Appl. Phys. 22, 2966-2972

Ng, H. W. (1995) "Dynamic analysis of AGR fuel assemblies", Computers \& Structures, Volume 54, Issue 2, 17 January 1995, Pages 339-350

Roscow, A. R., Skelton, J. \& Mclachlan, N. (2008) "Continuing Development of the Quarter Scale Rig to Support the Safe Performance of Graphite Reactor Cores", Securing the Safe Performance of Graphite Reactor Cores, $24^{\text {th }}$. 26 $6^{\text {th }}$ November 2008, Nottingham, UK

Steele, J.A., Martin, L.A., McArthur, S.D.J., Moyes, A.J., McDonald, J.R., Howie, D., Elrick, R. \& Yule, I.Y. (2003) "An intelligent system for interpreting the nuclear refuelling process within an advanced gas-cooled reactor" Proceedings of the Institution of Mechanical Engineers, Part A: Journal of Power and Energy, 217 (2). Pages 159-167

Wallace, C.J., West, G.M., Jahn, G.J., McArthur, S.D.J. Buckley, G. \& Towle, D., (2010) "Control Rod Monitoring of Advanced Gas Cooled Reactors", Seventh American Nuclear Society International Topical Meeting on Nuclear Plant Instrumentation, Control, and Human-Machine Interface Technologies, NPIC\&HMIT 2010, Las Vegas, Nevada, USA, $7^{\text {th }}-11^{\text {th }}$ November 2010

Ziver, K., Pain, C. C., Carter, J. N., de Oliveira, C. R. E., Goddard, A. J. H. \& Overton, R. S., (2004) "Genetic algorithms and artificial neural networks for loading pattern optimisation of advanced gas-cooled reactors", Annals of Nuclear Energy, Volume 31, Issue 4, March 2004, Pages 431-457 